This item was submitted to Loughborough's Institutional Repository by the author and is made available under the following Creative Commons Licence conditions.

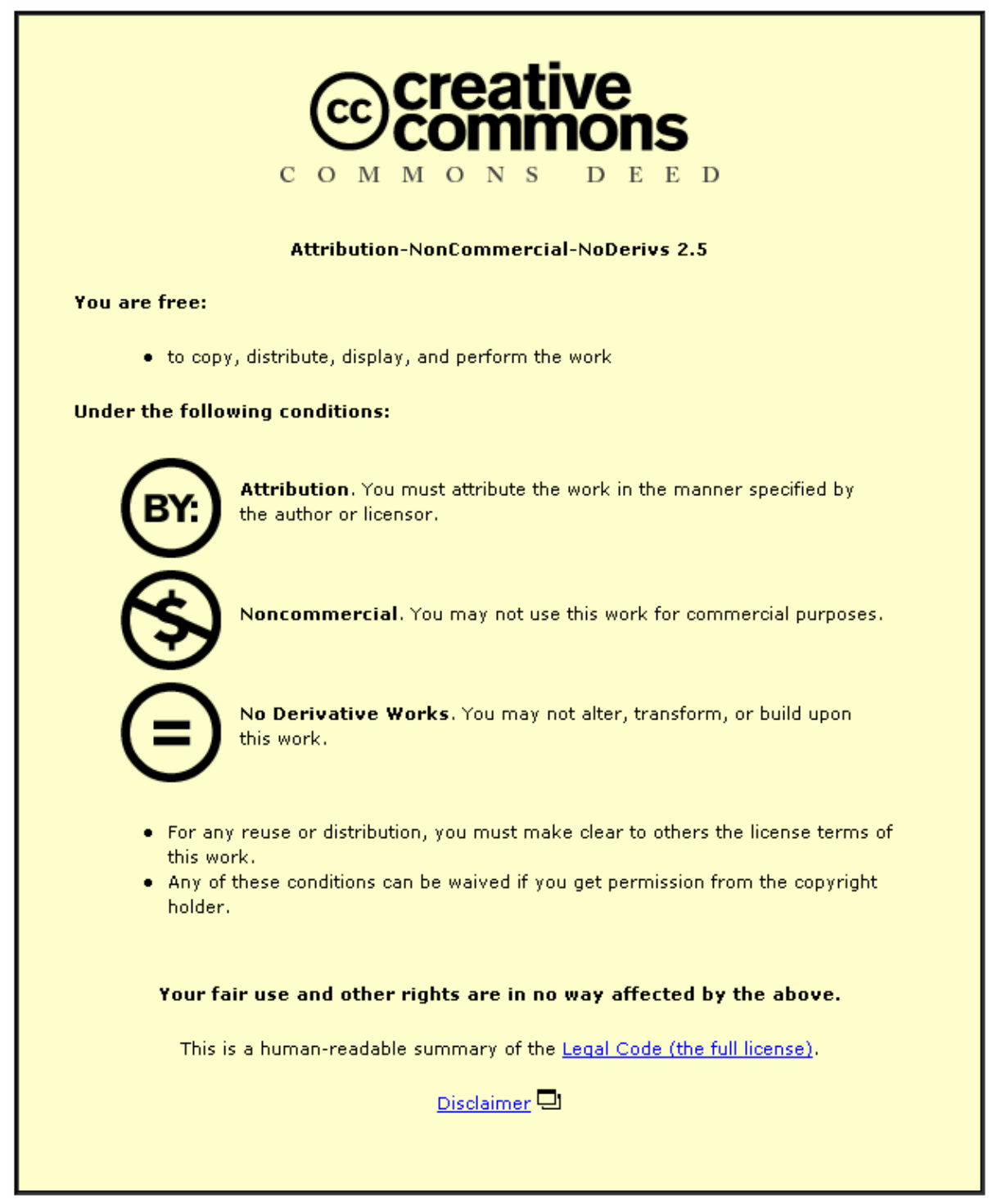

For the full text of this licence, please go to: http://creativecommons.org/licenses/by-nc-nd/2.5/ 


\title{
Chitin in a dual role as substrate for Streptomyces griseus and as adsorbent for streptomycin produced during fermentation.
}

\author{
Richard J. L. Meanwell and Gilbert Shama* \\ Department of Chemical Engineering, Loughborough University, Loughborough, \\ LEICS, LE11 3TU, UK.
}

\begin{abstract}
Streptomycin production from chitin by Streptomyces griseus was compared using two different types of bioreactor. The first was equipped with a combined U-shaped paddle and Rushton impeller. Also employed was a bioreactor of novel design in which the chitin was contained in a wire mesh basket that was totally submerged in a liquid salts medium. During operation the chitin was gently fluidised by air admitted into the basket. Fermentation was continued in both bioreactors until maximum antibiotic titres were achieved whereupon operation was interrupted to allow the streptomycin adsorbed to the chitin substrate to be extracted into $\mathrm{pH} 3.0$ buffer before continuing fermentation of the same batch of chitin a second time. At a chitin concentration of $10 \%(\mathrm{w} / \mathrm{v})$ the highest streptomycin yields $(c .5 .5 \mathrm{mg} / \mathrm{L})$ were obtained using the stirred bioreactor, however growth occurred more rapidly in the vertical basket bioreactor.
\end{abstract}

Keywords: Chitin; Streptomyces; Streptomycin; Stirred Bioreactors; Vertical Basket Bioreactor

\footnotetext{
*Corresponding author. Tel.: + 441509 222514; fax: + 441509223923

E-mail address; g.shama@Lboro.ac.uk
} 


\section{Introduction}

Chitin is an abundant biopolymer that occurs in the shells of crustaceans, the carapaces of insects and in the cell walls of fungi. Although many of these sources of chitin are too dispersed to be exploited, commercial fishing of crustaceans, and their subsequent processing, generates large quantities of chitin-rich waste [1]. This waste can be purified by either chemical or biological methods [2] to yield chitin that can be used in a number of applications within the food [3] medical and veterinary [4,5] or environmental protection industries [6].

One common factor in many of the more traditional applications to which chitin and the closely related partially deacetylated polymer, chitosan, have been put is based on the powerful chelating properties of these polymers. Both chitin and chitosan have been used to remove a diverse range of compounds from waste streams including heavy metals [7] and dyes [8,9]. Chitin has also been used in the microbial production of chitinases as fungal control agents $[10,11]$ and as a source of polyene antibiotics produced by a number of species of Monascus [12]. More recently, it was shown that a strain of Pseudomonas fluorescens cultured on chitin produced thermally stable antifungal compounds active against a number of phytopathogens [13].

Although the ability to utilise chitin is fairly widespread amongst both marine and terrestrial micro-organisms [14], nearly all species of the genus Streptomyces are chitinolytic [15]. The streptomycetes, are economically important because they produce a number of high value secondary metabolites including many antibiotics 
[16]. Streptomycin, the first aminoglycoside antibiotic discovered, is produced by Streptomyces griseus, and remains a useful weapon in the fight against the resurgence of tuberculosis [17].

Streptomycete fermentations are characterised by high broth viscosities owing to the filamentous nature of the organism, and the maintenance of adequate mixing and aeration in conventional stirred bioreactors is achieved by vigorous agitation. Operation under these conditions leads to high shear rates and can result in reduced yields, difficulties with subsequent downstream processing and even product degradation $[18,19]$. The incorporation of chitin, a solid, in culture media in conventional stirred bioreactors would result in additional stresses being imposed on the streptomycetes filaments due to the abrasive action of the chitin particles. Consequently, in investigating the cultivation of S. griseus on chitin for the production of streptomycin, we sought to employ bioreactors designed to minimise physical damage to the organism. We employed two different types of bioreactor; the first was a stirred bioreactor equipped with an impeller of special design. This comprised a combination of U-shaped paddle and Rushton impeller that was designed to be operated at low speed whilst maintaining the chitin in suspension. The second type of bioreactor investigated here was one of novel design and incorporated a static vertical basket in which the chitin was contained whilst being immersed in a salts medium. Air was passed directly into the basket so as to both provide oxygen to the culture of S. griseus, and to gently fluidise the chitin flakes.

In addition to serving as substrate, the chitin also acted as adsorbent for the streptomycin produced during fermentation. Because the chitin is solid and easily separated from the aqueous broth, it was possible to interrupt the fermentation so that 
adsorbed streptomycin could be recovered. Following this, the fermentation was allowed to proceed again before conducting a further cycle of streptomycin recovery.

\section{Materials and methods}

\subsection{Micro-organism \& Cultivation}

Streptomyces griseus (NCIMB 8136) was purchased in lyophilised form (NCIMB, Aberdeen, UK). Spores of the organism were maintained on sterile soil and were cultured on yeast malt extract agar (NCIMB Medium No. 29) at $28^{\circ} \mathrm{C}$ when required for bioreactor experiments. The liquid medium used for such experiments comprised flakes of chemically purified chitin (Sigma Aldrich plc, Poole, Dorset, UK) at concentrations specified in the text and the following salts per litre of distilled water; $\mathrm{K}_{2} \mathrm{HPO}_{4}, 0.76 \mathrm{~g} ; \mathrm{KH}_{2} \mathrm{PO}_{4}, 0.3 \mathrm{~g} ; \mathrm{MgSO}_{4}, 0.5 \mathrm{~g} ; \mathrm{FeSO}_{4}, 0.01 \mathrm{~g} ; \mathrm{ZnSO}_{4}, 0.0018 \mathrm{~g}$; $\mathrm{MnCl}_{2}, 0.0016 \mathrm{~g}$. The $\mathrm{pH}$ of the medium was adjusted to 6.5 and was autoclaved at $121^{\circ} \mathrm{C}$ for 20 minutes. Inoculum for bioreactors comprised $200 \mathrm{ml}$ of culture grown in the salts medium supplemented with $1 \%(\mathrm{w} / \mathrm{v})$ chitin for 96 hours in an incubatorshaker at $100 \mathrm{rpm}$ and $28{ }^{\circ} \mathrm{C}$.

\subsection{Streptomycin Determination}

The concentration of streptomycin was determined using a standard bioassay procedure [20] and was based on the inhibition of the growth of Bacillus subtilis (NCIMB 8054). The only variation introduced into the procedure was in the method 
of producing that the spores of B. subtilis [21]. Culture broths were first filtered through $0.2 \mu \mathrm{m}$ Whatman cellulose nitrate membrane filters (Fisher Scientific, Loughborough, Leics.) and subsequently using $2 \mathrm{kDa}$ membranes (Dow Danmark A/S, Nakskov, Denmark). Aliquots of filtered culture broths $(150 \mu \mathrm{L})$ were added to wells cut into agar plates which were incubated for 48 hours at $30^{\circ} \mathrm{C}$. Zones of inhibition were measured using Vernier callipers. All determinations were performed at least in duplicate. A standard calibration curve was obtained using aqueous solutions of streptomycin (Sigma Aldrich plc).

\section{$2.3 \mathrm{CO}_{2}$ Determination}

Gas samples were taken from the air exit line of the bioreactor using a gas-tight syringe and directly injected into a gas chromatograph (Model 104, Pye Unicam, Cambridge, UK) equipped with a thermal conductivity detector operated at $180^{\circ} \mathrm{C}$. The glass chromatography column was $6 \mathrm{~mm}$ diameter and $175 \mathrm{~cm}$ long and was packed with Molecular Sieve 5A (Phase Separations, Deeside, Clwyd, UK).

\subsection{Contamination Monitoring}

Samples of fermentation broth were routinely plated onto Tryptone Soy Agar (Oxoid, Unipath Ltd., Basingstoke, Hants) and Malt Extract Agar (Oxoid) and incubated at 30 and $25^{\circ} \mathrm{C}$ respectively and examined after 2 and 4 days for the presence of contamination. 


\subsection{Bioreactors}

\subsubsection{Stirred Bioreactor}

A standard stirred 2-litre glass bioreactor vessel (LH Engineering Co. Ltd., Stoke Poges, Bucks, UK) was used with a working volume of 1.5 litres. The air flowrate to the sparger was $100 \mathrm{ml} / \mathrm{min}$. Agitation was provided by an impeller that comprised a U-shaped paddle and a Rushton impeller of $5 \mathrm{~cm}$ diameter (Figure 1). The U-shaped paddle was $23 \mathrm{~cm}$ wide and $18 \mathrm{~cm}$ high and fabricated from stainless steel strips $1 \mathrm{~cm}$ wide and was operated at $55 \mathrm{rpm}$. After filling with medium, the bioreactor was autoclaved at $121^{\circ} \mathrm{C}$ for 40 minutes.

\subsubsection{Vertical Basket Bioreactor}

This comprised a cylindrical wire frame of height $105 \mathrm{~cm}$ and diameter $65 \mathrm{~cm}$ (Figure 2) fabricated around a hollow central support member and held in place by circular end plates. Wire mesh $(0.2 \mathrm{~mm}$ aperture $)$ was wound round the frame to create a chamber into which the chitin could be contained. The base plate was fabricated from sheet steel fitted with a wire mesh plate $1 \mathrm{~cm}$ above it. Air was admitted into the basket via the hollow central support. The air emerged from four $0.5 \mathrm{~mm}$ diameter holes at the base of the support and below the wire mesh and flowed upwards in the form of fine bubbles through the basket. The liquid working volume was 1.0 litre. An air flowrate of $100 \mathrm{ml} / \mathrm{min}$ was found to be sufficient to bring about gentle fluidisation of the chitin flakes contained in the chamber. In addition, two aerators were installed outside of the basket to both further aerate the liquid medium and to provide agitation; 
the flowrate to each of these aerators was $150 \mathrm{ml} / \mathrm{min}$. The total liquid volume was 1 litre. The mass of chitin flakes added to the basket was $35 \mathrm{~g}$. After filling with medium, the bioreactor was autoclaved at $121^{\circ} \mathrm{C}$ for 40 minutes.

\subsection{Recovery of Adsorbed Streptomycin from Chitin}

\subsubsection{Stirred Bioreactor}

At the termination of fermentation experiments the chitin was allowed to sediment and the culture broth drawn off. An equal volume of $\mathrm{pH} 3.0$ buffer (made by mixing $597 \mathrm{ml}$ citric acid $(0.1 \mathrm{M})$ with $153 \mathrm{ml}$ dibasic sodium phosphate $(0.2 \mathrm{M}))$ and diluting to $1500 \mathrm{ml}$ buffer was added. Extraction was allowed to proceed at ambient temperature for 24 hours in situ in the bioreactor at ambient temperature at an impeller speed of $55 \mathrm{rpm}$. The treated chitin was then added to the salts medium in the bioreactor and re-autoclaved as described above. The bioreactor was re-inoculated once the temperature of the medium had reached $28^{\circ} \mathrm{C}$.

\subsubsection{Vertical Basket Bioreactor}

At the termination of fermentation experiments the aqueous portion of the medium was pumped out of the bioreactor and replaced with an equal volume of sterile $\mathrm{pH} 3.0$ buffer solution. Extraction was then allowed to proceed for 24 hours under conditions of fluidisation. After extraction of the streptomycin the buffer was pumped out of the 
bioreactor and replaced with an equal volume of salts medium. Fermentation was allowed to proceed immediately without necessitating a second inoculation.

\section{Results}

\subsection{Stirred Bioreactor}

Figure 3 shows $\mathrm{CO}_{2}$ profiles with time for two fermentations performed in succession on the same batch of chitin in the stirred bioreactor with in each case an initial chitin concentration of $7.5 \% \mathrm{w} / \mathrm{v}$. In the first fermentation the $\mathrm{CO}_{2}$ peak occurs 48 hours earlier than in the subsequent one but in both cases the peak $\mathrm{CO}_{2}$ concentrations are within $5 \%$ of one another. For reasons given in the Discussion, antibiotic titres in Figure 4 and in all subsequent figures are reported as 'apparent streptomycin concentration'. The streptomycin titres (Figure 4) display a broadly similar pattern to one another, however, that for the first fermentation shows a slightly more pronounced peak occurring 100 hours after the $\mathrm{CO}_{2}$ maximum. The final streptomycin concentration reached at the end of the first fermentation at $2.5 \mathrm{mg} / \mathrm{L}$ is marginally greater than that achieved in the second fermentation $(2.3 \mathrm{mg} / \mathrm{L})$. The $\mathrm{pH}$ change over the entire time course of this and all subsequent fermentations never amounted to more than 0.4 units and suggests that the chitin provided some buffering capacity.

The use of the specially designed impeller enabled the chitin concentration to be increased to $10 \%(\mathrm{w} / \mathrm{v})$ without any settling out of the chitin or its accumulation in any of the 'dead zones' of the bioreactor. As for the fermentations described above, 
the same batch of chitin was subjected to two successive fermentations. $\mathrm{CO}_{2}$ profiles obtained under these conditions are shown in Figure 5. As for the previous fermentations, the profiles are very similar to one another and reach maximum concentrations at times comparable to those of Figure 4. Significantly, the $\mathrm{CO}_{2}$ peak also occurs earlier in the second fermentation. Streptomycin titres are compared in Figure 6. The curve for the first fermentation displays a peak of $2.2 \mathrm{mg} / \mathrm{L}$ at approximately 380 hours, whereas that for the second fermentation peaks at 340 hours before declining and rising again to a final value of $2.0 \mathrm{mg} / \mathrm{L}$. In both cases antibiotic titres were lower than those achieved at the lower chitin concentration of $7.5 \%$.

\subsection{Vertical Basket Bioreactor}

The procedure for conducting two successive fermentations with the same batch of chitin were similar to those for the stirred bioreactor but differed in that the chitin was not re-autoclaved after the first fermentation. Figure 7 shows the $\mathrm{CO}_{2}$ concentration against time profiles for $10 \%(\mathrm{w} / \mathrm{v})$ chitin fermentation using the vertical basket bioreactor. These are similar to one another, both reaching maxima at approximately 175 hours, significantly earlier than was the case for the stirred bioreactor. The maximum streptomycin titres were also similar to one another (Figure 8). The maximum titre reached in the first fermentation was $2.8 \mathrm{mg} / \mathrm{L}$ at 300 hours and 3.0 $\mathrm{mg} / \mathrm{L}$ at approximately 250 hours. Antibiotic titres were both greater than those obtained in the stirred bioreactor and were achieved in shorter times.

Table 1 shows streptomycin yields in the liquid medium, those recovered from the solid chitin and total yields. The former were obtained by multiplying streptomycin 
titres at the end of the fermentation by final liquid volume remaining in the bioreactor - the working volume of the stirred bioreactor was 1.5 litres and that of the vertical basket bioreactor was 1.0 litre. The streptomycin yields recovered from solid chitin were obtained by multiplying streptomycin titres in the extraction buffer at 24 hours by the volume of buffer employed. The stirred bioreactor clearly resulted in the production of greater amounts of streptomycin both in the liquid medium and adsorbed on the chitin. Although total yields are comparable at both chitin concentrations ( 7.5 and $10.0 \%$ ), the results of Table 1 suggest that the contribution made to total yield by the 'solid fraction' was greater at the higher chitin concentration. Both 'solid' and 'liquid' streptomycin yields are lower in the vertical basket bioreactor.

\section{Discussion}

Preliminary studies (not reported here) conducted using shake flasks had revealed the presence of anti-microbial compounds in the early phases of growth of S. griseus on chitin. These were compounds that inhibited the growth of the bioassay bacterium $(B$. subtilis) but were evidently not secondary metabolites as they were produced during the idiophase. GC and HPLC assays were employed in the early phases of fermentation in order to eliminate definitively the presence of streptomycin and whilst none was detected, the tests were too insensitive for all but the highest concentrations of streptomycin encountered in this work [22]. Further tests revealed that these anti- 
microbial compounds could be removed by filtering the culture broths through a 2 $\mathrm{kDa}$ membrane and therefore all samples taken during the course of fermentations for streptomycin determination by bioassay were filtered thus prior to testing by bioassay. However, Fourier Transform Infra Red (FTIR) analysis conducted on solid chitin remaining at the end of fermentation revealed the unambiguous presence of streptomycin [22]. As the quantity of solid chitin required for FTIR analyses was too large to permit the technique to be used routinely throughout the time course of fermentations, reliance had to be placed on the bioassay as the most sensitive method for detecting streptomycin. Under such conditions it was felt more appropriate to refer to 'apparent streptomycin' titres.

The presence of solid chitin flakes in the fermentation medium to which the growing streptomycete filaments became attached, prevented the direct determination of biomass concentration. Consequently, reliance had to be placed on $\mathrm{CO}_{2}$ concentration in the exit gas stream from the bioreactor as an indicator of growth. Unpublished work conducted with the strain of S. griseus used here in a medium devoid of chitin had shown that a close correlation existed between the concentration of biomass and the concentration of $\mathrm{CO}_{2}$ in the gas outlet from the bioreactor [22]. This correlation was important and confirmed the validity of using exit gas $\mathrm{CO}_{2}$ concentration as an indirect indication of microbial growth. $\mathrm{CO}_{2}$ evolution is commonly used as an indicator of growth and has been preferred to other indirect methods such as assays for cell wall components [23]. Work conducted to characterise the effects of agitation on cell lysis in Streptomyces clavuligerus also revealed concomitant increases in the concentrations of biomass and $\mathrm{CO}_{2}$ [18]. 
Peak $\mathrm{CO}_{2}$ evolution was achieved more rapidly in the vertical basket bioreactor than in the stirred bioreactor. This suggests that biomass growth was more rapid in the in the former (Figures $5 \& 7$ ). This was undoubtedly a reflection of the different shear regimes established in the two bioreactors, as mechanical stresses are known to influence not only the morphology of streptomycetes but to have the ability to induce cell lysis [18,24]. Evidently, the shears inside the basket were less intense than those in the stirred bioreactor.

The maximum yield of streptomycin achieved using the vertical basket bioreactor (2.8 $\mathrm{mg} / \mathrm{L})$ compare favourably with that achieved $(3.0 \mathrm{mg} / \mathrm{L})$ in a chitin-free medium containing yeast extract and malt extract [22]. Whilst growth was more rapid in the vertical basket bioreactor, total streptomycin titres were greater in the stirred bioreactor (Table 1). Convincing evidence has been accumulated to show that stresses of different types can enhance the production of secondary metabolites. The imposition of heat, salt or ethanol stresses stimulated antibiotic production by Pseudomonas fluorescens [25]. Similarly, greater yields of the antibiotic microcin were obtained when $E$. coli was cultured in a shake flask rather than in a rotating wall bioreactor intended to simulate conditions of microgravity [26].

The advantage offered by the vertical basket bioreactor configuration was that the 'draw and fill' process could be conducted with relative ease and without having to autoclave the chitin residue in between and without encountering subsequent contamination. 
Taken together these findings suggest that the performance of the vertical basket bioreactor might be improved still further. Conditions similar to those described here could be maintained to achieve rapid biomass growth during idiophase. Once optimal biomass concentration had been reached, the hydrodynamic environment inside the basket could be altered so as to increase the level of mechanical stress imposed on $S$. griseus in order to maximise streptomycin yield. The most direct way of achieving this would be to increase the air flowrate to the basket which would have the effect of increasing the fluidisation intensity. Moreover, future studies of this type would need to be conducted using industrial strains of S. griseus which produce very much higher yields of streptomycin [27] in order to establish whether a process based on the vertical basket bioreactor could compete with the conventional method used to produce streptomycin.

\section{References}

1. Gildberg A, and Stenberg EA. A new process for advanced utilisation of shrimp waste. Proc. Biochem. 2001, 36 ,809-812

2. Zakaria Z, Hall GM, and Shama G. Lactic acid fermentation of scampi waste in a rotating horizontal bioreactor for chitin recovery. Proc. Biochem. 1998, 33, 1-6

3. Shahidi F, Kamil J, Arachchi V, and Jeon Y. Food applications of chitin and chitosans, Trends Food Sci. Tech. 1999, 10, 37-51 
4. Sashiwa H, Aiba SI. Chemically modified chitin and chitosan as biomaterials.

Prog. Poly. Sci. 2004, 29,887-908

5. Senel S, and McClure SJ. Potential applications of chitosan in veterinary medicine Adv. Drug Del. Revs. 2004, 56, 1467-1480

6. Synowiecki J, and Al-Khateeb NA. Production, properties, and some new applications of chitin and its derivatives. Crit. Revs Food Sci. Nutr. 2003, 43, 145-171

7. Dao Z, Zhang LN, Zhou JP, and Guo SL. Development of a fixed-bed column with cellulose/chitin beads to remove heavy-metal ions. J. Appl. Poly. Sci. 2004, 94, 684691

8. Longhinotti E, Pozza F, Furlan L, Sanchez MDND, Klug M, Laranjeira MCM, and Favere VT. Adsorption of anionic dyes on the biopolymer chitin. J. Braz. Chem. Soc. $1998, \mathbf{9}, 435-440$

9. Blackburn RS. Natural polysaccharides and their interactions with dye molecules: Applications in effluent treatment. Env. Sci. Tech. 2004, 38, 4905-4909

10. Mahadevan B, and Crawford DL. Properties of the chitinase of the antifungal biocontrol agent Streptomyces lydicus WYEC108. Enz. Micro. Tech. 1997, 20, 489493 
11. Trejo-Estrada SR, Paszczynski A, and Crawford DL. Antibiotics and enzymes produced by the biocontrol agent Streptomyces violaceusniger YCED-9. J. Ind Micro. Biotech. 1998, 21, 81-90

12. Wang SL, Yen YH, Tsiao WJ, Chang WT, and Wang CL. Production of antimicrobial compounds by Monascus purpureus CCRC31499 using shrimp and crab shell powder as a carbon source. Enz. Micro. Tech. 2002, 31 337-344

13. Wang SL, Yen YH, Tzeng GC, and Hsieh C. Production of antifungal materials by bioconversion of shellfish chitin wastes fermented by Pseudomonas fluorescens K188. Enz. Micro. Techn. 2005, 36, 49-56

14. Warren, RAJ. Microbial hydrolysis of polysaccharides. Ann. Rev. Micro. 1996, 50, 183-212.

15. Schrempf, H. Recognition and degradation of chitin by streptomycetes, Antonie Van Leeuwenhoek Int. J. Gen. Mol. Micro. 2001, 79, 285-289.

16. Paradkar A, Trefzer A, Chakraburtty R, Stassi D. Streptomyces genetics: A genomic perspective. Crit. Revs Biotech. 2003, 23,1-27.

17. Humma LM. Prevention and treatment of drug-resistant tuberculosis Am. J. Health-Sys. Pharm. 1996, 53, 2291-2298. 
18. Roubos JA, Krabben P, Luiten RGM, Verbruggen HB, and Heijnen JJ. A quantitative approach to characterizing cell lysis caused by mechanical agitation of Streptomyces clavuligerus. Biotech. Prog. 2001, 17, 336-347

19. Liu BL, Kao PM, Tzeng YM, Feng KC. Production of chitinase from Verticillium lecanii F091 using submerged fermentation. Enz. Micro. Tech. 2003, 33, 410-415.

20. Anon., Official Method 962.14, Chapter 33, pp 41-3, Association of Official Analytical Chemists (AOAC) Official Methods of Analysis, $16^{\text {th }}$ Edition. Volume 2, 1998, AOAC International, Gaithersburg, MD., USA.

21. Harnulv BG, and Snygg BG. Heat-resistance of Bacillus subtilis spores at various water activities. J. Appl. Bacteriol. 1972, 35, $615-624$

22. Meanwell RJL. Streptomycin production from chitin using Streptomyces griseus, PhD Thesis, 2004, Loughborough University.

23. Desgranges C, Vergoignan C, Georges M, and Durand A, Biomass estimation in solid state fermentation: I. Manual biochemical methods. Appl. Micro. Biotech. 1991, 35, 200-205

24. Pinto LS, Vieira LM, Pons MN, Fonseca MMR, and Menezes JC. Morphology and viability analysis of Streptomyces clavuligerus in industrial cultivation systems. Bioproc. Biosys. Eng. 2004, 26, 177-184 
25. Nakata K, Yoshimoto A, and Yamada Y. Promotion of antibiotic production by high ethanol, high $\mathrm{NaCl}$ concentration, or heat shock in Pseudomonas fluorescens S272. Biosci. Biotech. Biochem. 1999, 63, 293-297

26. Gao Q, Fang A, Pierson DL, Mishra SK, and Demain AL. Shear stress enhances microcin B17 production in a rotating wall bioreactor, but ethanol stress does not, Appl. Micro. Biotech. 2001, 56, 384-387

27. Butler, PR, Brown, M, and Oliver, SG. 1996. Improvement of antibiotic titres from Streptomyces bacteria by interactive continuous selection. Biotech. Bioeng. 49, $185-196$. 


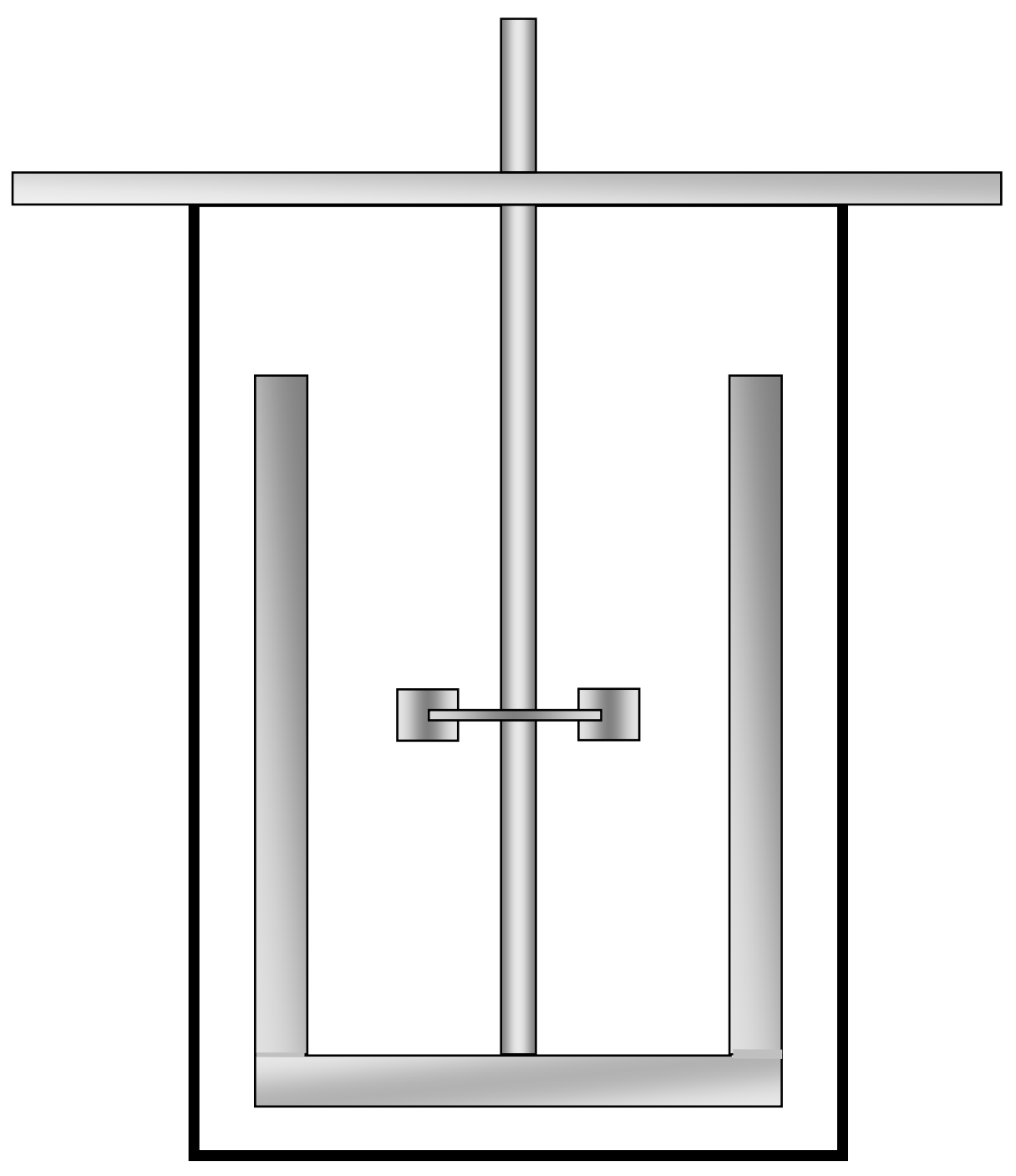

Figure 1 Impeller Arrangement for Stirred Bioreactor 


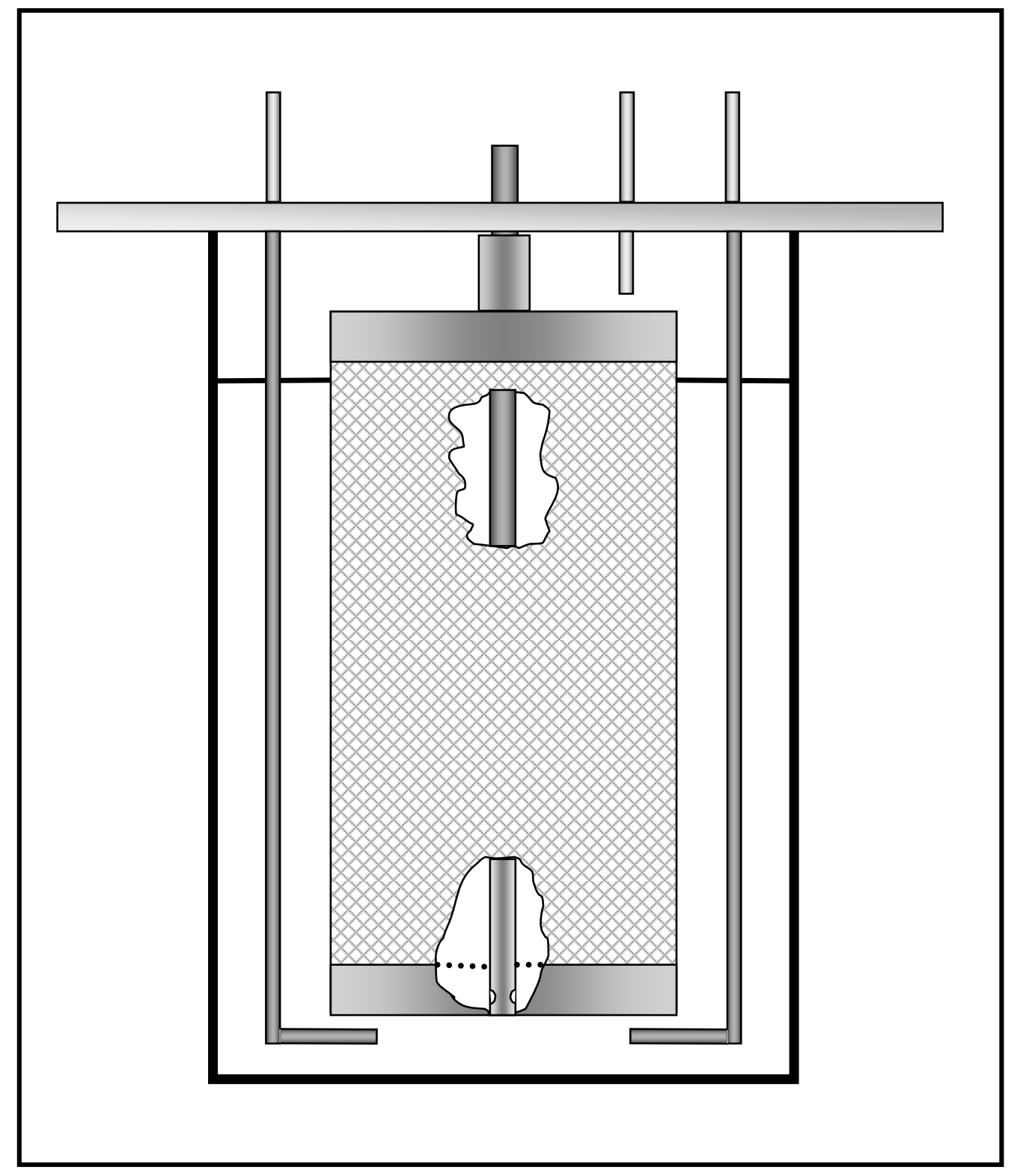

Figure 2 Vertical Basket Bioreactor 


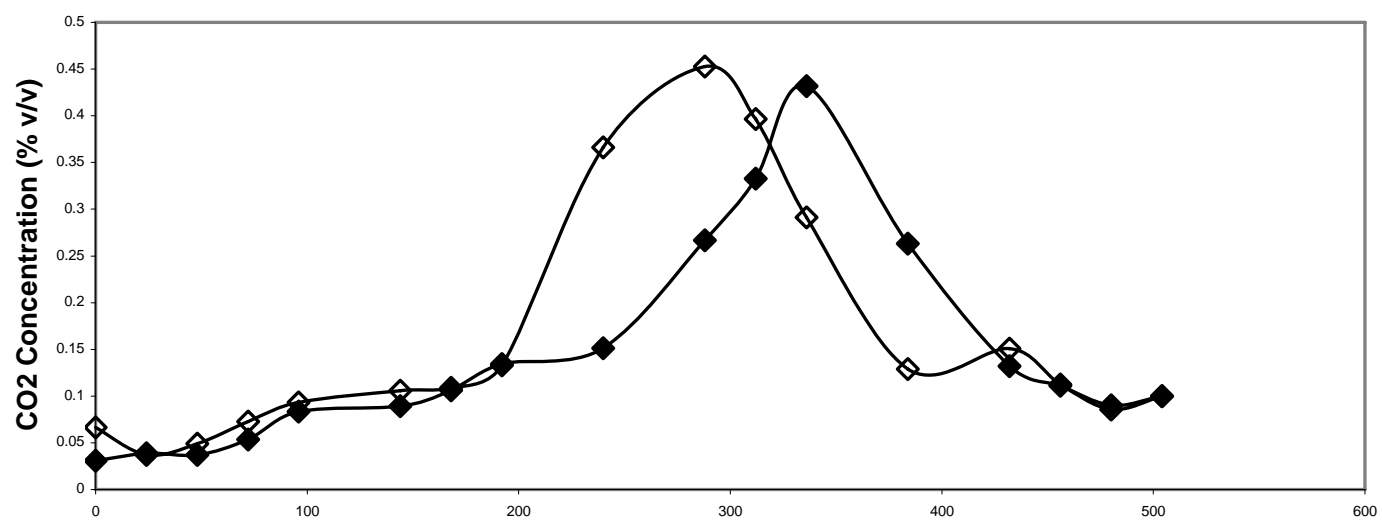

Figure $3 \mathrm{CO}_{2}$ Profiles during successive fermentation of chitin (7.5\%) in a stirred bioreactor $\longrightarrow 7.5 \%$ First Experiment $\longrightarrow$-7.5\% Second Experiment

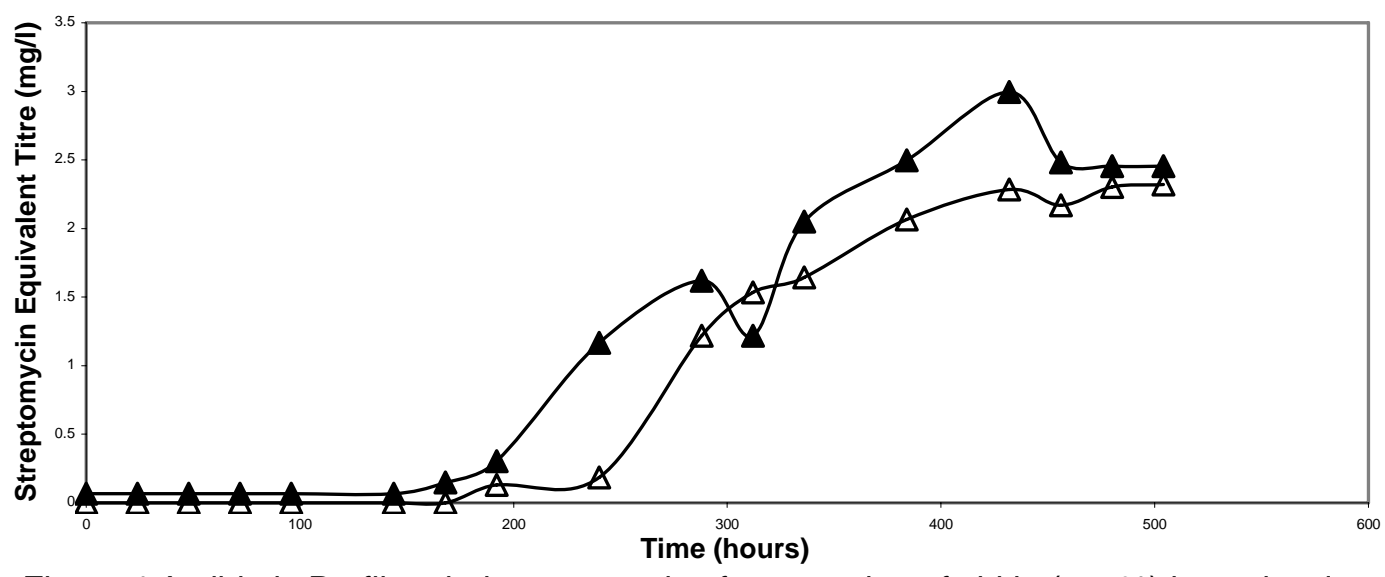

Figure 4 Antibiotic Profiles during successive fermentation of chitin (7.5\%) in a stirred bioreactor

$\longrightarrow$-7.5\% First Experiment $\longrightarrow$-7.5\% Second Experiment

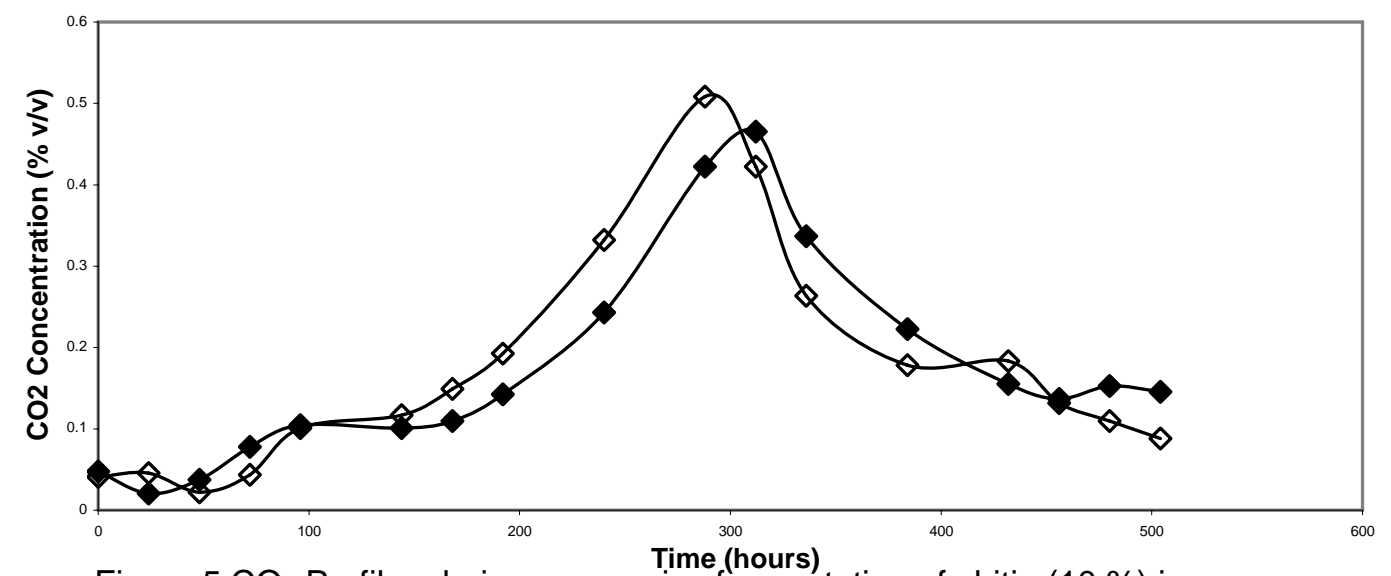

Figure $5 \mathrm{CO}_{2}$ Profiles during successive fermentation of chitin (10\%) in a stirred bioreactor 


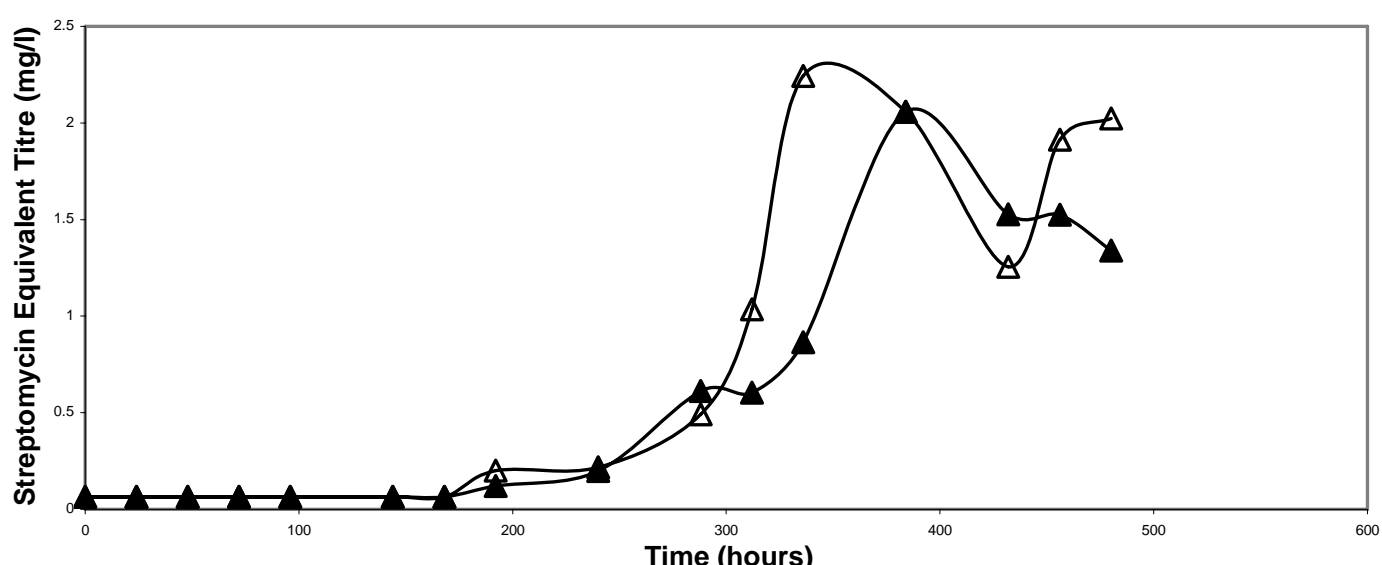

Figure 6 Antibiotic Profiles during successive fermentation of chitin (10\%) in a stirred bioreactor

$\longrightarrow$-10\% First Experiment $\longrightarrow 10 \%$ Second Experiment

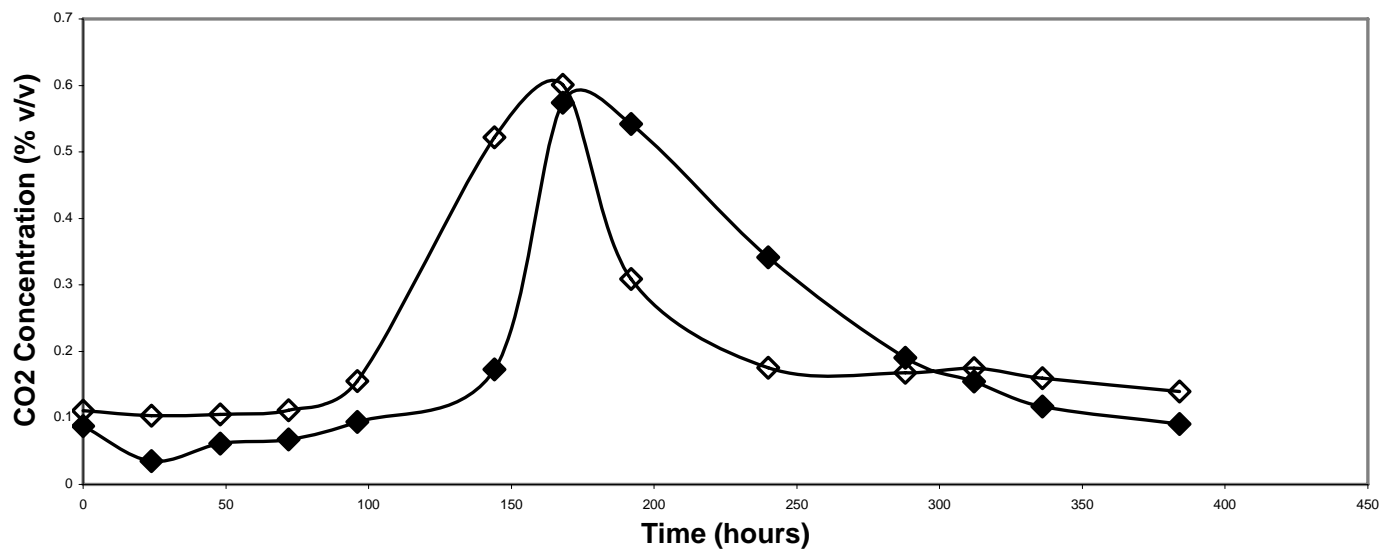

Figure $7 \mathrm{CO}_{2}$ Profiles during successive fermentation of chitin (10\%) in a Vertical Basket bioreactor

$\longrightarrow$ First Experiment $\longrightarrow$ - Second Experiment

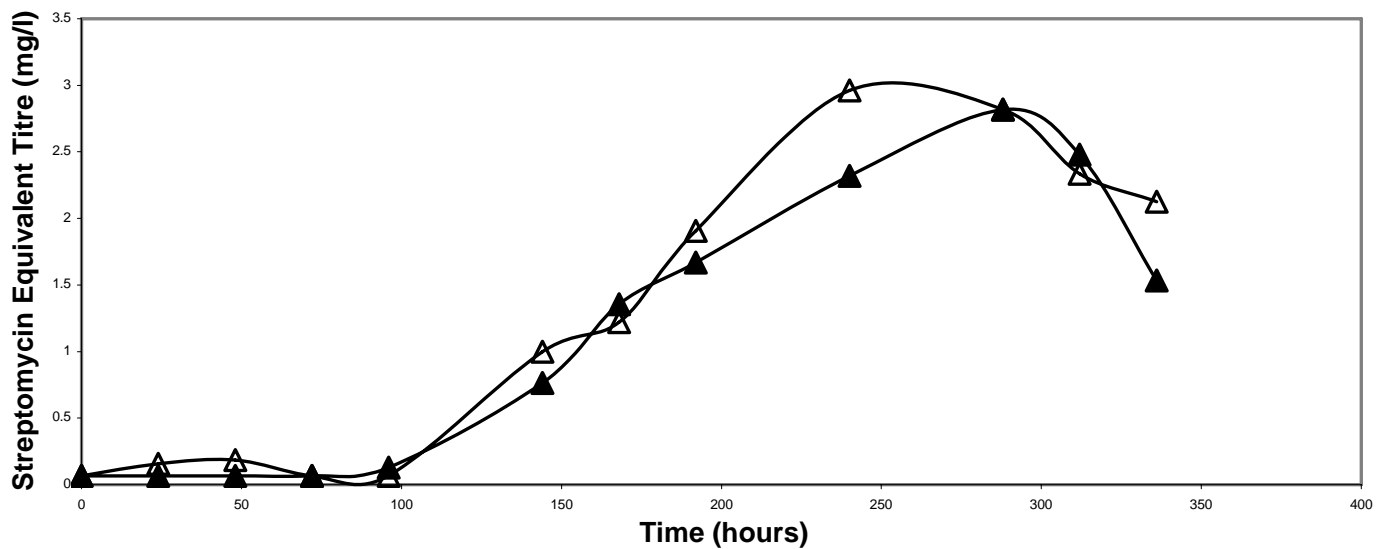

Figure 8 Antibiotic Profiles during successive fermentation of chitin (10\%) in a Vertical Basket bioreactor 


\begin{tabular}{|c|c|c|c|c|c|c|}
\hline \multirow[b]{2}{*}{ Bioreactor } & \multicolumn{4}{|c|}{ Stirred } & \multicolumn{2}{|c|}{ Vertical Basket } \\
\hline & $1^{\text {st }}$ Ferm & $2^{\text {nd }}$ Fer1 & ${ }^{\text {st }}$ Fern & ${ }^{\text {nd }}$ Ferm & $1^{\text {st }}$ Fern & $2^{\text {nd }}$ Ferm \\
\hline $\begin{array}{l}\text { Chitin Conc. } \\
(\mathrm{mg} / \mathrm{L})\end{array}$ & 7.5 & 7.5 & 10.0 & 10.0 & 10.0 & 10.0 \\
\hline $\begin{array}{l}\text { Streptomycin Yield } \\
\text { (Liquid, mg) }\end{array}$ & 3.48 & 4.49 & 3.36 & 3.09 & 2.82 & 2.96 \\
\hline $\begin{array}{l}\text { Streptomycin Yield } \\
\text { (Solid, mg) }\end{array}$ & 1.56 & 1.37 & 2.25 & 2.46 & 1.68 & 1.65 \\
\hline $\begin{array}{l}\text { Total Streptomycin } \\
\text { Yield (mg) }\end{array}$ & 5.04 & 5.86 & 5.61 & 5.55 & 4.50 & 4.61 \\
\hline
\end{tabular}

Table 1. Streptomycin Yields Obtained in Stirred and Vertical Basket Bioreactors 